\title{
Estilos de vida y cumplimiento de las Guías Alimentarias Chilenas: resultados de la ENS 2016-2017
}

\section{Lifestyles and adherence to the Chilean Dietary Guidelines: results of the Chilean National Health 2016-2017 Survey}

\begin{abstract}
RESUMEN
Cumplir las recomendaciones incluidas en las Guías Alimentarias permite un acercamiento hacia un estilo de vida saludable. El objetivo de este estudio fue caracterizar los estilos de vida de la población chilena y su asociación al cumplimiento de 5 recomendaciones de las Guías Alimentarias Basadas en Alimentos (GABA) chilenas. Se utilizaron datos de la Encuesta Nacional de Salud 2016-2017. Los participantes fueron divididos en 4 grupos: aquellos que no cumplieron con ninguna recomendación, los que cumplían una, los que cumplían con 2 y los que cumplían con 3 o más recomendaciones saludables, estableciendo a este último como grupo de referencia. El 43,3\% de la muestra no cumplió ninguna de las recomendaciones incorporados en esta investigación. De los que cumplían a lo menos 3, el 35,9\% presentó un estado nutricional normal, el $38,9 \%$ reportó nunca haber fumado y el $82 \%$ se auto percibía como saludable. Además, los que cumplían las recomendaciones, presentaron niveles más altos de actividad física. Personas que incorporan y aplican las recomendaciones de las GABA, tienden a presentar conductas de vida más saludable. Estrategias comunicacionales efectivas y formativas en educación alimentaria sobre estas recomendaciones son claves para prevenir el acelerado y temprano crecimiento de las patologías crónicas. Palabras clave: Educación alimentaria y nutricional (DeCS); Estilo de vida saludable; Guías alimentarias.
\end{abstract}

\footnotetext{
ABSTRACT

Compliance with dietary guidelines is essential for adoopting a healthy lifestyle. This study aimed to characterise the lifestyles of the Chilean population according to the fulfilment of $5 \mathrm{re}-$ commendations of the Food-Based Dietary Guidelines (GABA in Spanish) established in Chile according to WHO definition. Data from the 2016-2017 National Health Survey were used. Participants were divided into four groups: those who did not meet any recommendations, those who met one, those who met two and those who met three or more (reference group) GABA health recommendations. $43.3 \%$ of the sample did not meet any of the recommendations incorporated into this study. Of those who fulfilled at least three recommendations, $35.9 \%$ had a normal nutritional status, 38.9\% reported never having smoked, and $82 \%$ perceived themselves as healthy. In
}

\begin{abstract}
Claudia Troncoso-Pantoja ${ }^{7}$, Fabián Lanuza ${ }^{2,3}$,
María Adela Martínez-Sanguinetti ${ }^{4}$, Ana María Leiva ${ }^{5}$, Karina Ramírez-Alarcón ${ }^{6}$, Miquel Martorell ${ }^{6,7}$, Ana María Labraña ${ }^{6}$, Solange Parra-Soto ${ }^{8,9}$, Nicole Lasserre-Laso ${ }^{10}$, Gabriela Nazar ${ }^{7,11}$, Carlos Celis-Morales ${ }^{8,9,12,13}$, Fanny Petermann-Rocha ${ }^{8,9^{*}}$, en representación del grupo ELHOC.
\end{abstract}

1. Centro de Investigación en Educación y Desarrollo (CIEDE-UCSC), Departamento de Salud Pública, Facultad de Medicina, Universidad Católica de la Santísima Concepción, Concepción, Chile

2. Biomarkers and Nutrimetabolomics Laboratory, Department

of Nutrition, Food Sciences and Gastronomy, CIBER Fragilidad y

Envejecimiento Saludable (CIBERFES), Faculty of Pharmacy and Food Sciences, University of Barcelona, Barcelona, Spain.

3. Centro de Epidemiología Cardiovascular y Nutricional (EPICYN),

Facultad de Medicina, Universidad de La Frontera, Temuco, Chile. 4. Instituto de Farmacia, Facultad de Ciencias, Universidad Austral de Chile, Valdivia, Chile.

5. Instituto de Anatomía, Histología y Patología, Facultad de Medicina, Universidad Austral de Chile, Valdivia, Chile.

6. Departamento de Nutrición y Dietética, Facultad de Farmacia, Universidad de Concepción, Concepción, Chile.

7. Centro de Vida Saludable, Universidad de Concepción, Concepción, Chile. 8 Institute of Health and Wellbeing, University of Glasgow, Glasgow, United Kingdom.

9. BHF Glasgow Cardiovascular Research Centre, Institute of Cardiovascular and Medical Sciences, University of Glasgow, Glasgow, United Kingdom. 10. Escuela de Nutrición y Dietética, Facultad de Salud, Universidad Santo Tomás, Chile.

11. Departamento de Psicología, Facultad de Ciencias Sociales, Universidad de Concepción, Concepción, Chile.

12. Centro de Investigación en Fisiología del Ejercicio (CIFE), Universidad Mayor, Santiago, Chile.

13. Laboratrio de Rendimiento Humano, Grupo de Estudio en Educación, Actividad Física y Salud (GEEAFyS), Universidad Católica del Maule, Talca, Chile.

*Dirigir correspondencia a: Fanny Petermann-Rocha, BHF Glasgow Cardiovascular Research Centre, Institute of Cardiovascular and Medical Sciences, College of Medical, Veterinary and Life Sciences University of Glasgow, Glasgow, G12 8TA, United Kingdom. Email : f.petermann-rocha.1@research.gla.ac.uk

Este trabajo fue recibido el 10 de diciembre de 2019. Aceptado con modificaciones: 08 de abril de 2020. Aceptado para ser publicado: 07 de mayo de 2020. 
addition, those who fulfilled the recommendations presented higher levels of physical activity (1343.5 MET/min/day [95\% Cl: 1049.9 - 1637.2]). From the results, it is inferred that people who incorporate and apply GABA recommendations tend to present healthier life behaviours. Consequently, effective and formative communication strategies in food education on these recommendations are crucial to preventing a fast and early growth of chronic diseases.

Keywords: Dietary guidelines; Food and nutrition education; Healthy lifestyle.

\section{INTRODUCCIÓN}

En las últimas décadas, la población de Latinoamérica y en especial la chilena, ha presentado una fuerte transición en su perfil demográfico y epidemiológico, observándose una nueva pirámide poblacional, caracterizada por un aumento de la población de personas mayores por sobre el promedio de la población general, en especial, la población infantil'. Esta situación ha significado un incremento de la prevalencia de patologías crónicas asociadas al envejecimiento de la población; tales como obesidad, diabetes mellitus tipo 2, enfermedades cardiovasculares y diversos tipos de cáncer ${ }^{2,3}$. Asociado a lo anterior, se han generado cambios socioeconómicos, culturales y ambientales, que han impactado directamente en los estilos de vida de la población, incluidos sus patrones alimentarios ${ }^{4,5}$. Ejemplo de ello, es el caso de la población chilena, que ha privilegiado el consumo de alimentos altos en energía, en azúcares simples y grasa, como también bajos en fibra dietética ${ }^{6}$. Esta transformación de hábitos alimentarios y la presencia de un ambiente obesogénico, han favorecido el incremento de enfermedades crónicas y el aumento de la malnutrición por exceso del peso corporal ${ }^{7,8}$.

En este contexto, se han desarrollado en Chile materiales gráficos para realizar acciones educativas en alimentación y nutrición. En el año 1948, se utilizó la primera clasificación que incluyó cuatro grupos de alimentos, graficados en un plato dividido en cuatro, según aportes de nutrientes ${ }^{9}$. En el año 1955, el Ministerio de Salud de Chile reconoció esta clasificación, que estuvo vigente hasta el año 1984. A partir del año 1985, y hasta el año 1986, se adoptó la clasificación en cinco grupos de alimentos según nutrientes aportados; para volver a una imagen gráfica, que incluyó tres grupos de alimentos a partir del año $1989^{9}$. No obstante, las primeras Guías Alimentarias para la población chilena surgen en el año 1997, en conjunto con la Pirámide Alimentaria, en donde se estableció la variedad, proporción y porciones de los alimentos a consumir ${ }^{10}$. Desde el año 2013, están vigentes las Guías Alimentarias Basadas en Alimentos (GABA). Este grupo de recomendaciones que se basan en mensajes educativos adaptados desde la evidencia científica sobre alimentación y actividad física constituye un instrumento educativo, que, a través de 11 recomendaciones, busca promover y educar a la población acerca del consumo de alimentos saludables y práctica de actividad física, con el fin de prevenir enfermedades crónicas y obesidad en la población mayor de 2 años $^{11,12}$. A lo anterior, y a nivel global, su adecuada interpretación y utilización es una herramienta que permite fortalecer la educación alimentaria ${ }^{13}$, con experiencias exitosas en talleres educativos como los desarrollados por Rodríguez et al ${ }^{14}$ en población de Brasil o Dosil-Bermúdez en escolares de España ${ }^{15}$.

Pese a los esfuerzos desplegados por educar a la población, la Encuesta Nacional de Salud 2016-2017 (ENS 2016-2017) identificó que más del $74 \%$ de la población presentaba, entre otras enfermedades crónicas, sobrepeso u obesidad, situación preocupante y que cuestiona, entre otros factores, la calidad de la alimentación de la población chilena ${ }^{3}$. En este contexto y reconociendo la importancia de una alimentación saludable en la prevención de enfermedades crónicas y promoción de un buen estado de salud, el objetivo del estudio fue caracterizar los estilos de vida de la población chilena y su asociación al cumplimiento de 5 recomendaciones de las Guías Alimentarias Basadas en Alimentos (GABA) chilenas.

\section{MATERIALES Y METODOS Diseño del estudio}

Se realizó un estudio descriptivo de corte transversal. La muestra seleccionada comprendió a los participantes adultos de la Encuesta Nacional de Salud, desarrollada entre agosto del 2016 a marzo del 2017 (ENS 2016-2017). Esta Encuesta, fue un estudio de prevalencia realizado en viviendas particulares, constituyendo una muestra nacional, probabilística, estratificada y multietápica de 6.233 personas mayores de 15 años, con representatividad nacional, regional y del área urbana/rural. Para ponderar la muestra a población nacional, se aplicaron los factores de expansión sugeridos por la ENS 2016-2017. Por lo tanto, la muestra expandida incluida en este estudio representó a 14.489 .700 personas. El protocolo fue aprobado por el Comité de Ética de la Escuela de Medicina de la Pontificia Universidad Católica de Chile. Todos los participantes firmaron un consentimiento informado ${ }^{3}$.

\section{Grupos según cumplimiento de GABA}

Se consideró el auto-reporte de 5 recomendaciones de las GABA y que fueron incluidas en la ENS 2016-2017: el consumo de 5 porciones de frutas y verduras al día; consumo de 3 porciones de lácteos al día; ingesta de 6 vasos o más de agua al día; consumo de legumbres 2 veces por semana y consumo de pescado 2 veces a la semana. La información fue recolectada a través de auto-reporte.

A partir de los mensajes seleccionados, se crearon 4 grupos, clasificando a los sujetos según el grado de cumplimiento de las recomendaciones establecidas en los mensajes saludables: no cumple ninguna recomendación, cumple con una recomendación, cumple con 2 recomendaciones y cumple con 3 o más recomendaciones, estableciendo a este último como grupo de referencia.

\section{Variables sociodemográficas}

Las variables sociodemográficas consideradas para este estudio fueron: edad, sexo, zona geográfica de residencia (urbano/ rural), nivel de escolaridad clasificado en tres categorías (básica 
$<8$ años, media: 8 a 12 años, y técnica-universitaria $>12$ años) e ingreso económico en pesos chilenos (CLP), clasificado en tres categorías (bajo $<\$ 250.000$; medio $\$ 250.000$ a $\$ 450.000$ $y$ alto $>\$ 450.000)$.

\section{Mediciones antropométricas y metabólicas}

El peso corporal fue medido a través de una balanza electrónica digital (OMRON HN 289) y la talla, mediante cinta métrica metálica, escuadra y cinta adhesiva para afirmar en una muralla o puerta, ambos procedimientos se realizaron sin zapatos y ropa ligera. La medición del perímetro de cintura (PC) fue realizada con una cinta métrica inextensible, usando los protocolos establecidos ${ }^{3}$. Con estas mediciones se obtuvo el estado nutricional, el que fue clasificado en base a los puntos de corte del índice de masa corporal (IMC), según los criterios de la Organización Mundial de la Salud (OMS, bajo peso: IMC $<18,5 \mathrm{~kg} / \mathrm{m}^{2}$; normo peso: $18,5-24,9 \mathrm{~kg} / \mathrm{m}^{2}$; sobrepeso: $25,0-$ $29,9 \mathrm{~kg} / \mathrm{m}^{2}$ y obesidad: $\left.\geq 30,0 \mathrm{~kg} / \mathrm{m}^{2}\right)$, mientras que la obesidad abdominal fue definida como $P C \geq 88 \mathrm{~cm}$ para mujeres y $P C \geq$ $102 \mathrm{~cm}$ en hombres ${ }^{16}$. Estos puntos de corte fueron aplicados a toda la población estudiada.

El diagnóstico de síndrome metabólico (SM) fue realizado por la presencia de 3 o más componentes de riesgo, según ENS 2016-20177. La hipertensión arterial (HTA) se confirmó con dos mediciones de presión arterial estándares en cada brazo, separados en 30 segundos y en días distintos. Se consideró estado HTA a valores promedio de presión arterial sistólica $\geq$ $140 \mathrm{mmHg}$ o presión arterial diastólica $\geq 90 \mathrm{mmHg}$. Finalmente, la diabetes mellitus tipo 2 fue estimada por cualquiera de las situaciones diagnósticas determinadas en la Guía Clínica de Diabetes, esto es, glicemia en ayunas $\geq 126 \mathrm{mg} / \mathrm{dl} \mathrm{o} \geq 200 \mathrm{mg} /$ dl post-carga de glucosa ${ }^{18}$.

\section{Estilos de vida}

Los datos asociados con los estilos de vida, como el tabaquismo, horas de sueño $(<7$ horas/día; 7-9 horas/día y $>9$ horas/día) y auto-reporte de alimentación (agua, frutas y verduras), salud y bienestar (malo, regular o bueno), se obtuvieron mediante la aplicación de cuestionarios validados en la ENS 2016-2017. El consumo de sal se determinó mediante análisis de excreción de sodio en la orina, a través de la fórmula de Tanaka ${ }^{19}$. Los niveles de actividad física fueron determinados con el cuestionario Global Physical Activity Questionnaire $(\mathrm{GPAQ})^{20}$. Los niveles de sedentarismo fueron determinados mediante el auto-reporte de tiempo destinado a actividades que involucren estar sentado o reclinado durante el tiempo libre o de trabajo, a través del mismo cuestionario. Se consideró como punto de corte para inactividad física un gasto energético $<600 \mathrm{METs} /$ minutos/semana, según las recomendaciones de la OMS y especificaciones en la Guía de Análisis de GPAQ ${ }^{20}$.

\section{Análisis estadístico}

Los datos de caracterización de la población estudiada son presentados como promedios para variables continuas o como proporción para variables categóricas con su respectivo intervalo de confianza (95\% IC).
Para identificar si el promedio de las variables de salida (adiposidad medida a través del PC o IMC, estilos de vida, alimentación, actividad física y tiempo sedente) presentaban una tendencia significativa a disminuir o aumentar según cumplimientos de las guías alimentarias seleccionadas, se realizaron análisis de regresión lineal para muestras complejas, corregidos por el factor de expansión de la ENS 2016-2017. Debido a que este trabajo es un estudio descriptivo, no se realizaron ajustes por variables de confusión a las variables estudiadas.

Para todos los análisis se utilizó el programa STATA MP v15 y el comando de "svyset" para muestras complejas. Todos los resultados fueron estimados utilizando muestras expandidas acorde a los factores de expansión sugeridos por la ENS 20162017. El nivel de significancia fue definido como $p<0,05$.

\section{RESULTADOS}

En la tabla 1 se presentan las características generales de la población, según los cuatro grupos clasificados de acuerdo con el cumplimiento de las recomendaciones de las GABAS seleccionadas. Se destaca que el $43,3 \%$ de los participantes no cumplieron con ninguna de las recomendaciones alimentarias estudiadas y solo el 4,7\% cumplía con 3 o más recomendaciones. Los grupos con menos o ningún cumplimiento de las recomendaciones alimentarias, tenían menor nivel educacional y menor ingreso económico, en comparación al grupo que cumplía 3 o más recomendaciones saludables.

En la figura 1 se observa el cumplimiento de las GABA según indicadores antropométricos. En relación con el peso corporal, el grupo con mayor cumplimiento de las recomendaciones saludables presentó un menor promedio de peso corporal $(75,7 \mathrm{~kg}$ [95\% IC: 72,$1 ; 79,3])$ que los grupos que cumplían con 1 ó 2 recomendaciones alimentarias. Sin embargo, este fue superior al grupo que no cumplió ninguna recomendación (74,4 kg [95\% IC: 73,3; 75,5]). Al analizar el IMC y PC, se observó que el grupo de referencia presentó un menor IMC y PC en comparación a los grupos que cumplían solo con una, dos o ninguna recomendación alimentaria. No obstante, estas diferencias no fueron estadísticamente significativas, $p$ (tendencia): 0,422 (Figura 1).

En la figura 2, se aprecia la relación entre diversos estilos de vida y cumplimiento de las GABA. Se observa que el grupo de referencia presentó un mayor nivel de actividad física total (1343,5 MET/min/día [95\% IC: 1049,9; 1637,2]) en comparación a los otros grupos; sin embargo, la tendencia no fue significativa. Al relacionar el cumplimiento de las GABAS con las horas de sueño y actividad física, los valores fueron heterogéneos entre los grupos de estudio, sin diferencias significativas, destacándose la actividad física vigorosa y de transporte, donde el grupo de referencia tuvo menor puntuación que los grupos con menor o ningún cumplimiento de las recomendaciones alimentarias y una mejor percepción de salud que los otros dos grupos (Figura 2).

Finalmente, y en relación al consumo de agua, frutas y verduras, se observó una tendencia estadísticamente significativa ( $p$ (tendencia) <0,001) en el grupo de referencia (Figura 3). 
Tabla 1. Características generales según cumplimiento 5 recomendaciones de las guías alimentarias (GABA).

\begin{tabular}{|c|c|c|c|c|}
\hline & $\begin{array}{l}\text { No cumple ninguna } \\
\text { recomendación }\end{array}$ & $\begin{array}{c}\text { Cumple } 1 \\
\text { recomendación }\end{array}$ & $\begin{array}{c}\text { Cumple } 2 \\
\text { recomendaciones }\end{array}$ & $\begin{array}{l}\text { Cumple } 3 \text { o más } \\
\text { recomendaciones }\end{array}$ \\
\hline \multicolumn{5}{|l|}{ Sociodemográficas } \\
\hline n (\%) & $2.693(43,3 \%)$ & $2.278(36,6 \%)$ & $960(15,4 \%)$ & $286(4,6 \%)$ \\
\hline Población extendida & 682.464 & 2.258 .944 & 5.123 .558 & 6.424 .734 \\
\hline Edad (años) & $42,6[41,4 ; 43,8]$ & $44,2[42,8 ; 45,5]$ & $43,5[41,6 ; 45,4]$ & $41,3[37,3 ; 45,4]$ \\
\hline \multicolumn{5}{|l|}{ Zona geográfica (\%) } \\
\hline Rural & 10 & 12 & 12,7 & 10 \\
\hline Urbana & 90 & 88 & 87,3 & 90 \\
\hline Años de estudio & $10,8[10,5 ; 11,1]$ & $10,7[10,5 ; 11,0]$ & $11,3[10,6 ; 11,8]$ & $12,1[11,2 ; 13,0]$ \\
\hline \multicolumn{5}{|l|}{ Nivel educacional (\%) } \\
\hline Técnico-universitaria & 28,2 & 24,4 & 30 & 38,5 \\
\hline \multicolumn{5}{|l|}{ Nivel de ingreso (\%) } \\
\hline Bajo & 26,2 & 28 & 26 & 12 \\
\hline Medio & 31,9 & 32,4 & 33,3 & 37 \\
\hline Alto & 41,9 & 39,6 & 40,7 & 51 \\
\hline \multicolumn{5}{|l|}{ Antropométricas } \\
\hline Peso corporal (kg) & $74,4[73,3 ; 75,5]$ & $76,6[75,3 ; 77,9]$ & $76,3[74,7 ; 77,8]$ & $75,7[72,1 ; 79,3]$ \\
\hline Talla corporal (m) & $1,62[1,61 ; 1,63]$ & $1,63[1,62 ; 1,64]$ & $1,62[1,61 ; 1,64]$ & $1,66[1,64 ; 1,68]$ \\
\hline $\mathrm{IMC}\left(\mathrm{kg} / \mathrm{m}^{2}\right)$ & $28,3[27,9 ; 28,7]$ & $28,7[28,4 ; 29,1]$ & $28,9[28,5 ; 29,4]$ & $27,6[26,3 ; 28,9]$ \\
\hline \multicolumn{5}{|l|}{ Estado nutricional (\%) } \\
\hline Actividad física total & $1.137,8$ & $1.166,6$ & $1.254,8$ & $1.343,5$ \\
\hline (MET/min/día) & {$[1.005,3 ; 1.270,3]$} & {$[1.047,5 ; 1.285,8]$} & {$[1.090,0 ; 1.419,5]$} & {$[1.049,9 ; 1.637,2]$} \\
\hline Actividad física de transporte (min/día) & $98,5[83,6 ; 113,3]$ & $102,8[89,9 ; 115,6]$ & $114,6[93,1 ; 136,1]$ & $87,3[72,6 ; 101,9]$ \\
\hline \multirow[t]{2}{*}{ Actividad física moderada (min/día) } & 244,7 & 212,2 & 215,5 & 229,7 \\
\hline & {$[216,5 ; 272,9]$} & {$[189,8 ; 234,6]$} & {$[183,1 ; 248,0]$} & {$[172,1 ; 287,3]$} \\
\hline \multirow[t]{2}{*}{ Actividad física vigorosa (min/día) } & 228,5 & 205,1 & 207,0 & 172,5 \\
\hline & {$[197,4 ; 259,5]$} & {$[179,0 ; 231,1]$} & {$[172,7 ; 241,4]$} & {$[120,2 ; 224,8]$} \\
\hline Inactividad física (\%) & 28,4 & 24,1 & 23,1 & 16,2 \\
\hline Tiempo sedente (hora/día) & $3,6[3,4 ; 3,8]$ & $3,3[3,0 ; 3,5]$ & $3,3[2,9 ; 3,7]$ & $3,5[2,8 ; 4,2]$ \\
\hline Consumo de sal (g/día) & $9,1[8,9 ; 9,3]$ & $9,1[8,9 ; 9,3]$ & $9,2[8,9 ; 9,5]$ & $8,9[8,4 ; 9,5]$ \\
\hline Promedio horas de sueño día & $7,6[7,5 ; 7,7]$ & $7,5[7,3 ; 7,6]$ & $7,8[7,6 ; 7,9]$ & $7,5[7,3 ; 7,8]$ \\
\hline \multicolumn{5}{|l|}{ Horas de sueño (\%) } \\
\hline$<7$ horas/día & 22,3 & 25,9 & 16,9 & 24,9 \\
\hline 7-9 horas/día & 51,8 & 51,2 & 54,4 & 53,1 \\
\hline$>9$ horas/día & 25,9 & 22,9 & 28,7 & 22,0 \\
\hline \multicolumn{5}{|l|}{ Auto-reporte salud y bienestar (\%) } \\
\hline Malo & 2,7 & 1,3 & 1,6 & 2,4 \\
\hline
\end{tabular}




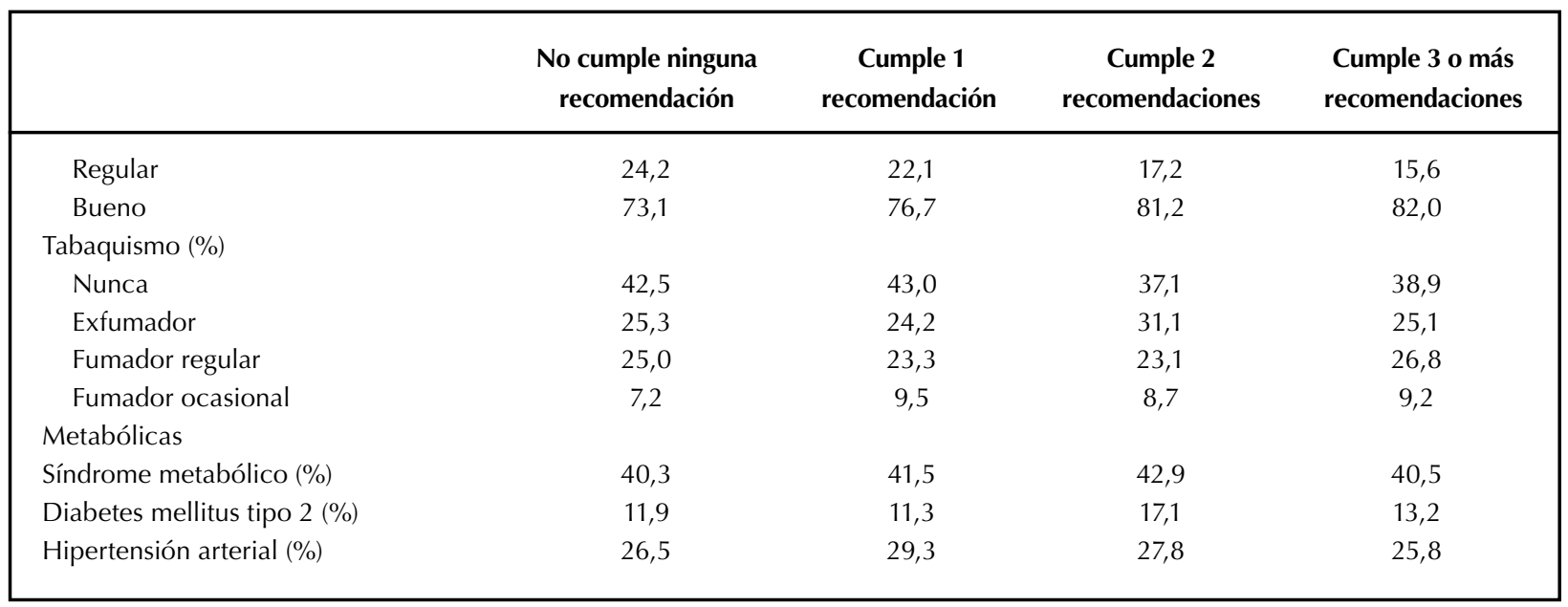

IMC: índice de masa corporal; MET: Metabolic Equivalents.

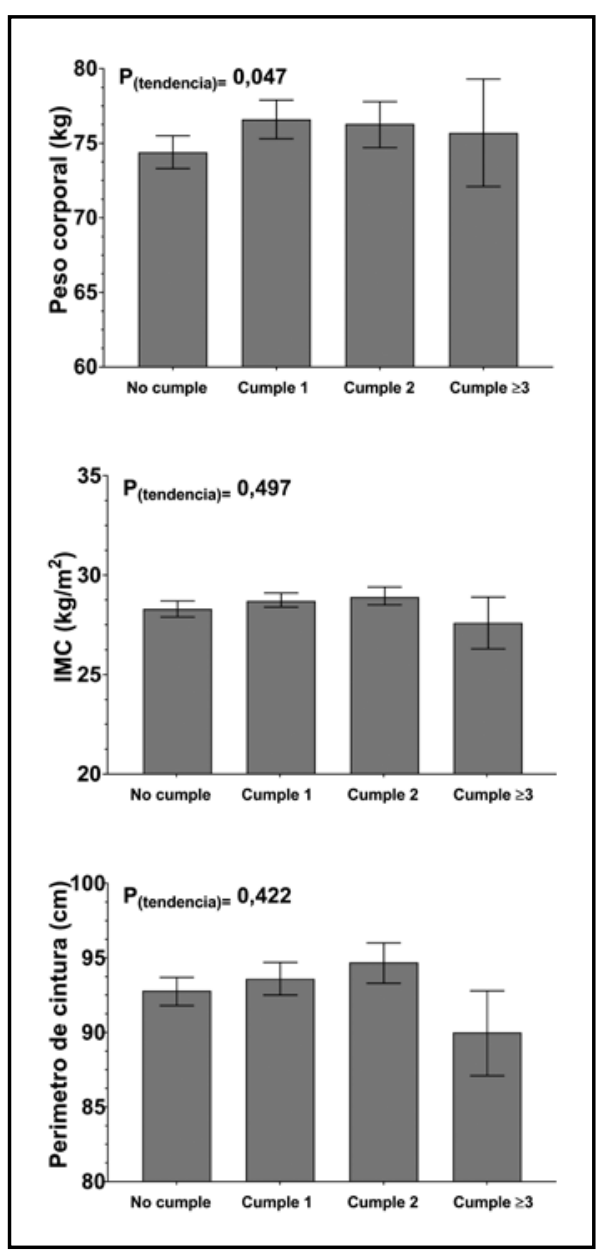

Figura 1: Cumplimiento de las recomendaciones de las GABA según indicadores antropométricos.

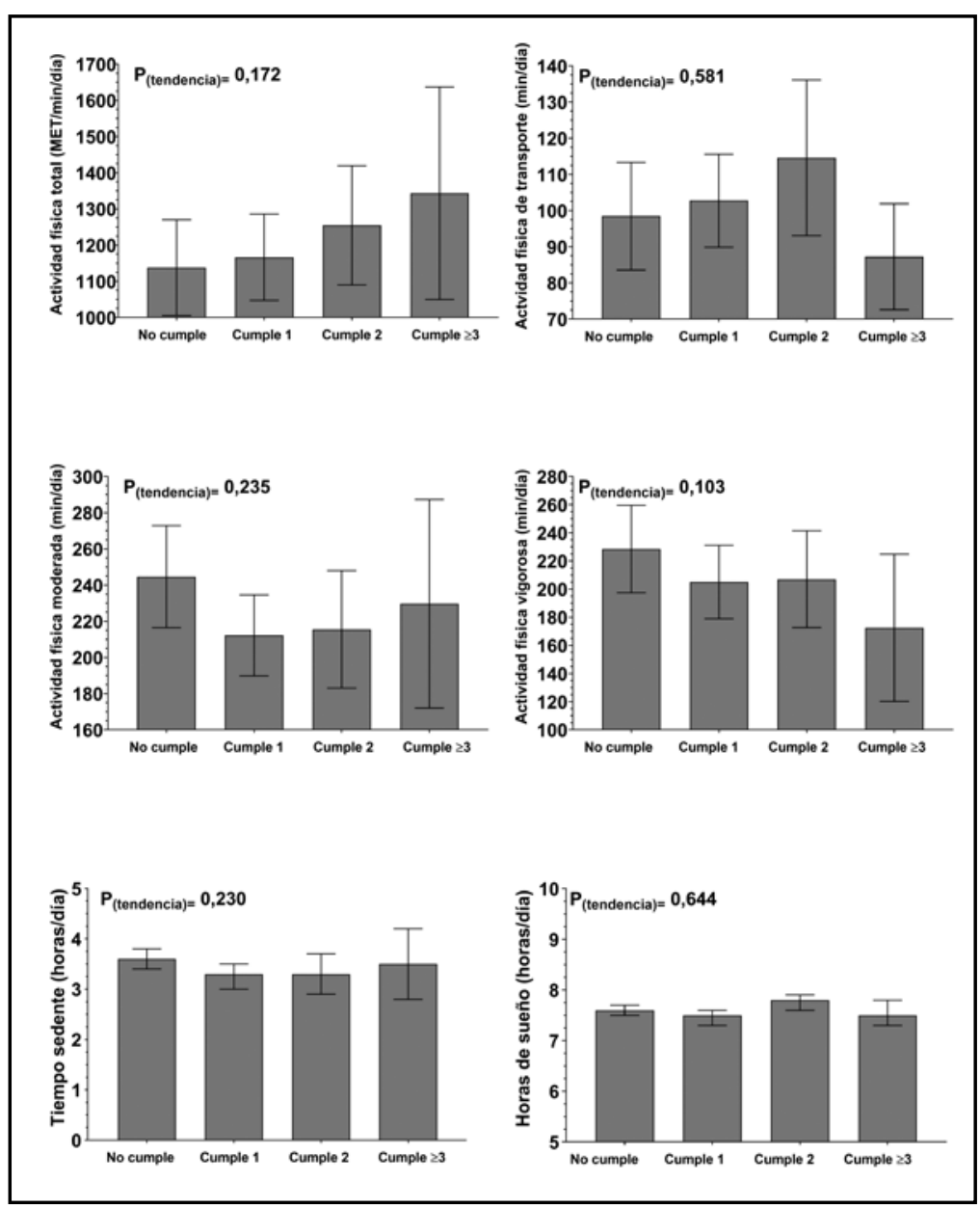

Figura 2: Cumplimiento de las recomendaciones de las GABA, según estilos de vida. 

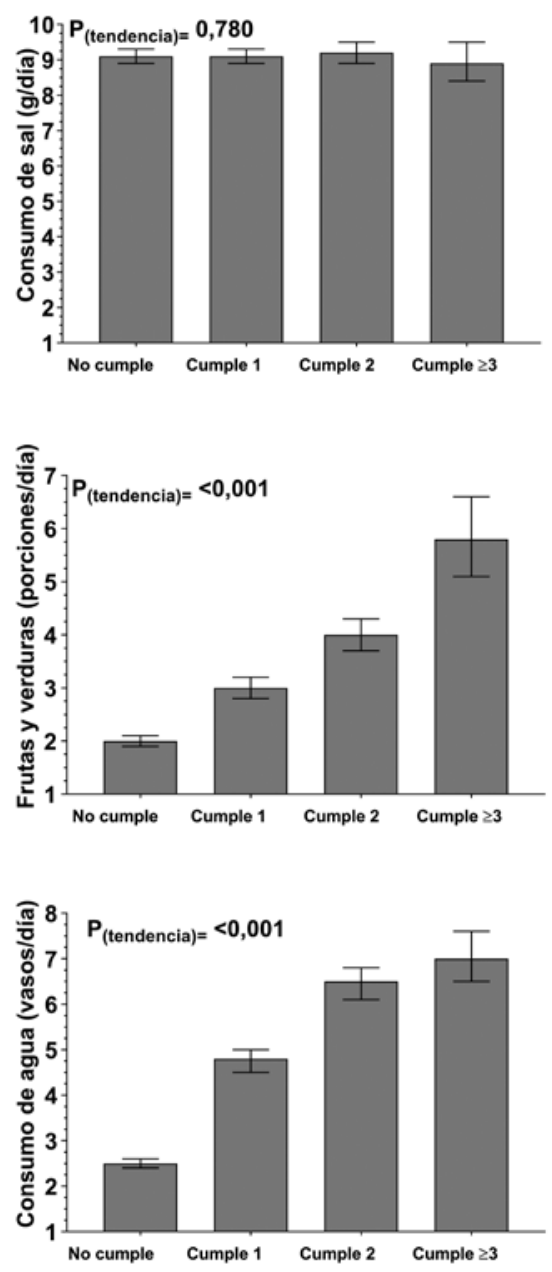

Figura 3: Cumplimiento de las recomendaciones de las GABA para conductas alimentarias.

\section{DISCUSIÓN}

Los resultados evidenciaron un bajo grado de cumplimiento de las 5 recomendaciones de las GABA estudiadas en este trabajo, desatacándose que, el 43,3\% de la población participante no cumplía con ninguna de las 5 recomendaciones de las GABA analizadas; por cada aumento en el cumplimiento de una recomendación, existió una tendencia significativa a aumentar el consumo de frutas, verduras y agua.

Existen limitados estudios en nuestro país que evalúen de forma integral estilos de vida según cumplimiento de recomendaciones alimentarias saludables de las GABA, por lo que, este estudio aporta evidencia importante en esta materia. La Encuesta Nacional de Consumo Alimentario publicada el año 2014 también evidenció un reducido cumplimiento de las GABA en la población participante, destacándose un bajo consumo de leguminosas (25\%), lácteos $(23 \%)$ y pescados $(17 \%)$, entre otros alimentos ${ }^{21}$. Otros estudios realizados en poblaciones de distintas edades también han evidenciado el bajo cumplimiento de las recomendaciones saludables contenidas en estas guías. Los autores Crovetto et $\mathrm{a}^{22}$, en un estudio en univeritarios chilenos, concluyen que la población estudiada presentaba un inadecuado consumo de nutrientes críticos, y consecuentemente un escaso cumplimiento de las recomendaciones de las guías alimentarias. Por otra parte, Barrón et $\mathrm{al}^{23}$ midieron en personas mayores sus hábitos alimentarios y actividad física, a través de cuestionario basado en las GABA, identificando entre sus resultados un consumo excesivo de sal y un acotado consumo de agua de manera diaria, lo que se aleja de las recomendaciones saludables de las GABA. Esta realidad también ha sido observada por otros autores a nivel internacional como, por ejemplo, Gille et $a^{24}$ en Suiza y Morínigo-Martínez en población del Paraguay ${ }^{25}$. Este último estudio expone que el $38 \%$ de la población paraguaya cumplían con las Guías Alimentarias del país, valores que se acercan a los encontrados en esta investigación.

La baja adherencia a las recomendaciones para una alimentación y vida saludable, hacen infructuosos los esfuerzos desplegados por el equipo de salud en las actividades de promoción o prevención en salud, identificándose causales dependientes del personal sanitario y también de los propios usuarios ${ }^{26}$. Dentro de los principales obstáculos identificados están la manera en que se recibe la educación por parte de los agentes de salud, la falta de compromiso para modificar hábitos o conductas de salud y un limitado acompañamiento de las redes sociales ${ }^{27}$. En nuestro estudio se compararon los participantes que seguían tres o más recomendaciones de las GABA, con aquellos que no acataban ninguna recomendación, observándose que estos últimos presentaron estilos de vida poco saludables, con mayor consumo de alcohol, menor ingesta de agua, frutas y verduras, además de presentar una menor realización de actividad física, lo que permite entrelazar la importancia de una alimentación saludable con factores condicionantes en el estado de salud y de vida.

Las recomendaciones 1 y 2 de las GABA consideran el desarrollo de actividad física para el mejoramiento de los estilos de vida ${ }^{10}$. Nuestros resultados señalan que el grupo que cumple con a lo menos tres de las recomendaciones de las GABA, presenta una mayor actividad física total y menor porcentaje de inactividad física; sin embargo, posee menor actividad física de transporte que podría explicarse, entre otros factores, por el acceso a vehículos y transporte motorizado que disminuyen un transporte activo. En la actualidad, no se concibe el hecho de que la alimentación saludable se centren solo en el comer y nutrirse de manera sana, sino también en el gasto energético que realiza una persona de manera diaria. En México ${ }^{28}$, Argentina $^{29}$ y 
también en nuestro país ${ }^{10}$, el desarrollo de actividad física es parte importante de las recomendaciones de las Guías Alimentarias, debido a que la alimentación saludable y el desarrollo de actividad física se complementan mutuamente como componentes esenciales en la mantención de estilos de vida saludables ${ }^{30}$, además de fomentar el control de patologías crónicas y la malnutrición por exceso, existiendo estudios clásicos ${ }^{31}$ y actuales ${ }^{32}$ que ratifican esta condición.

En nuestro estudio se observó que el IMC, PC y la subsecuente obesidad abdominal, se presenta con una alta prevalencia, pero destaca una menor proporción en el grupo que cumple 3 o más recomendaciones alimentarias, respecto a los que cumplen menos o ninguna recomendación. Existe suficiente evidencia nacional e internacional que relaciona una mala calidad de alimentación con malnutrición por exceso $^{33,34,35}$ y que, las Guías Alimentarias, se alzan como una herramienta educativa eficaz para mejorar o mantener estilos de vida saludables ${ }^{36}$.

El uso de Guías Alimentarias se ha expandido a nivel global, como un instrumento útil y necesario para la difusión de recomendaciones saludables en la población general. La revisión liderada por Herforth et $\mathrm{al}^{37}$ reconoce aspectos transversales a las Guías Alimentarias de diversos países, como el estar direccionadas a comunicar una orientación dietética local, considerar una organización por grupos de alimentos y la entrega de recomendaciones que se articulan a las concedidas por la OMS. Se considera que las GABA, son una estrategia de comunicación y educación que puede presentar un impacto real en la calidad de vida de la población, aunque el conocerlas, no es condicionante para cumplirlas o desarrollar comportamientos de salud positivos ${ }^{38}$. Sin embargo, su estrategia de uso es la que podría ser reconsiderada por los encargados en su implementación, al valorar la entrega de los mensajes de manera más pertinente y lúdica a la población objetivo. Una experiencia exitosa fue la realizada por Olivares et $\mathrm{al}^{39}$ en adolescentes chilenos entre 11 y 14 años. Utilizaron la red social WhatsApp, además de Tecnologías de Información y Comunicación (TICs), como videos y audios, para mantener o mejorar los estilos de vida saludable, usando las recomendaciones de las GABA. Entre los resultados, las autoras señalan que los participantes centran su comprensión de alimentación saludable en los mensajes de la GABA y preparaciones culinarias caseras.

\section{Fortalezas y limitaciones}

Como limitantes de este estudio, se pueden identificar la inclusión de solo cinco recomendaciones de las GABA en la ENS 2016-2017, lo que limita el análisis completo de las recomendaciones saludables de las GABA. El auto-reporte de las respuestas entregadas por los y las participantes también es una limitante en el estudio, originado desde el posible sesgo de memoria o un inadecuado mensaje verbal por parte del participante. Además, el diseño utilizado para la investigación, que impide establecer una relación de causa y efecto entre las recomendaciones y su cumplimiento. Como fortaleza, permite disponer de datos representativos de la población en estilos de vida saludables que responden a la realidad chilena y que las variables, peso corporal, talla y PC fueron recolectadas siguiendo protocolos estandarizados.

\section{CONCLUSIÓN}

Se reconoce una asociación entre los estilos de vida y el cumplimiento de las Guías Alimentarias Chilenas, identificando para esta investigación un bajo cumplimiento de las recomendaciones de las GABA. Lo anterior, unido a otros factores de riesgo, como la falta de actividad física, se confirman como un antecedente del incremento de las enfermedades crónicas no trasmisibles y consecuente deterioro de la calidad de vida de la población chilena. Sin embargo, también se considera que los participantes que siguen al menos tres recomendaciones de las GABA, presentaron una mayor probabilidad de presentar un estado nutricional y un PC en rangos de normalidad, además de presentar mayores niveles de actividad física y consumir mayor cantidad de frutas, verduras y agua.

Para lograr que la población aumente el grado de cumplimiento de las recomendaciones de las GABA, y adopten conductas de alimentación saludables, es necesario que se elaboren o potencien las estrategias integrales de difusión de sus mensajes saludables a todos los sectores de la población.

El etiquetado de los alimentos establecido en nuestro país ${ }^{40}$, avanzó en la divulgación de información saludable al público, regulando con mensajes de advertencia para conductas de riesgo nutricional, estimulando así la reducción de nutrientes críticos en los alimentos y normalizando la propaganda de estos últimos. No obstante, aún falta hacer propios estos esfuerzos intersectoriales en el país, para que la población le dé un sentido al autocuidado y se adopte un estilo de vida saludable. Sumado a lo anterior, se deben potenciar espacios seguros para la realización de actividad física.

Para lograr lo anterior, se requiere apoyo político y de recursos para ejecutar las distintas estrategias dirigidas a la población, como también es necesario realizar más estudios para la evaluación del cumplimiento de las GABA, en distintos grupos de la población.

\section{BIBLIOGRAFÍA}

1. Ortega-González D. Aging and treatment to the elderly in Chile: A Path of persistent inequality. Sophia Austral. 2018; 22: 223246.

2. OPS/OMS. Health Report of the Americas +2017. Organización Panamericana de la Salud/ Organización Mundial de la Salud, 2017.

3. MINSAL. National Health Survey 2016-2017. Ministerio de Salud, Gobierno de Chile, 2017.

4. Leiva AM, Petermann-Rocha F, Martínez-Sanguinetti MA, et al. Association of a healthy lifestyle index with cardiovascular risk factors in Chileans adults. Rev Med Chile. 2018; 146: 1405-1414. 
5. Egger G, Dixon J. Beyond obesity and lifestyle: a review of 21st century chronic disease determinants. Biomed Res Int. 2014; 2014: 731685.

6. Mattes R, Foster GD. Food environment and obesity. Obesity (Silver Spring). 2014; 22: 2459-2461.

7. Bray GA, Kim KK, Wilding JPH, et al. Obesity: a chronic relapsing progressive disease process. A position statement of the World Obesity Federation. Obes Rev. 2017; 18: 715-723.

8. Aguilar-Farias N, Miranda-Marquez $S$, Sadarangani $K P$, et al. Results from Chile's 2018 Report Card on Physical Activity for Children and Youth. J Phys Act Health. 2018; 15: S331-S332.

9. Hospital Dr. Exequiel González Cortés. Dietary guidelines for Chile. Medwave. 2002; 2: e2269.

10. Olivares, S. Zacarías I, Gonzalez CG, Villalobos E. Development and validation process of food-based dietary guidelines for the Chilean population. Rev Chil Nutr. 2013; 40: 262-268.

11. Olivares-Cortés S, Zacaría-Hasbún I, González-González CG, Fonseca-Morán L, Mediano-Stoltze F, Pinheiro-Fernandes AC et al. Design and validation of image for dissemination and implementation of Chilean dietary guidelines. Nutr Hosp. 2015; 32: 582-589.

12. Aranceta-Bartrina J, Arija-Val V, Maíz-Aldaur E, Martínez-de Victoria Muñoz E, Ortega-Anta RM, Pérez-Rodrigo $C$ et al. Food guidlines for the Spanish population (SENC, diciembre 2016); la nueva pirámide de la alimentación saludable. Nutr Hosp. 2016; 33: 1-48.

13. Vega M, Ejeda-Manzanera M, Caballero_armenta $M$, CuberoJuánez J, Ortega-Navas C. The Dietary Guidelines as didactic material in teacher training: análisis and application. Rev Complut. Educ. 2017; 28: 145-164.

14. Rodrigues $M$, Santos $A$, da Silva $D$, Falcão $O$, Mendes $R$. Nutritional knowledge after conducting nutritional education activities between children and adolescents sportspople. Pensar Prat. 2016; 19: 56-67.

15. Dosil-Bermúdez A. Pilotage of a healthy food workshop given to schoolars by a healthcare professional. Cad Aten Primaria. 2019; 25: 22-27.

16. Moreno M. Definition and classification of obesity. Rev Med Clin Condes. 2012; 23: 124-28.

17. Centro Universidad Católica - Surveys and longitudinal studies. National Health Survey ENS 2016 - 2017 [Chile]: Facultad de Medicina Pontificia Universidad Católica; 2016.

18. MINSAL. Clinical guide. Type 2 Diabetes Mellitus. Clinical Guide Series Minsal, 2010.

19. Tanaka T, Okamura T, Miura K, Kandowaski T, Ueshima H, Nakagawa $\mathrm{H}$ et al. A simple method to estimate populational 24-h urinary sodium and potassium excretion using a casual urine specimen. J Hum Hypertens. 2002; 16: 97-103.

20. Armstrong T, Bull F. Development of the World Health Organization Global Physical Activity Questionnaire (GPAQ). J Public Health. 2006; 14: 66-70.

21. Minsal. Universidad de Chile. National Survey of Food Consumption. Final report. 2014. Universidad de Chile.

22. Crovetto $M$, Figueroa B, González L, Jeira A, Ramírez $N$. Dietary guidelines and compliance in college students, Valparaíso, 2013, Chile. Rev Chil Nutr. 2015; 42: 164-172.

23. Barrón V, Rodríguez A, Chavarría P. Eating habits, nutritional status and lifestyle among active seniors in the city of Chillan, Chile. Rev Chil Nutr 2017; 44: 57-62.
24. Morínigo-Martínez M, Samudio M. Stages of the behavioral model of life styles in adults proposed by food guides of Paraguay. Mem. Inst. Investig Cienc Salud. 2015; 13: 41-48.

25. Gille D, Bütikofer U, Chollet M, Schmid A, Altintzoglou T, Honkanen $P$ et al. Nutrition behavior of the middle-aged and elderly: Compliance with dietary recommendations of the food pyramid. Clin Nutr. 2016; 35: 638-644.

26. Arrebola-Vivas E, López-Plaza B, Koester-Weber T, BermejoLópez L, Palma-Milla S, Lisbona-Catalán A et al. Predictor variables for low adherence to a lifestyle modification program of overweight treatment in primary health care. Nutr Hosp. 2013; 28: 1530-1535.

27. Oliva-Chávez OH, Fragoso-Díaz S. Fast food intake: the power of good food. RIDE. 2013; 4: 176-199.

28. Benvecchio A, Fernández-Gaxiola AC, Plazas M, KauferHorwitz M, Pérez AB, Rivera JA. Food and Physical Activity Guidelines in the context of overweight and obesity in the Mexican population. Intersistemas, S.A. de C.V. México, 2015.

29. Ministerio de Salud de la Nación. Food guides for the argentine population. Buenos Aires, 2016.

30. Nazar G, Stiepovic J, Bustos C. Latent class analysis of lifestyle behavior among Chilean university students. Int J Health Promot Educ. 2019; 57: 98-111.

31. Pan XR, Li GW, Hu YH, Wang JX, Yang WY, An ZX et al. Effects of diet and exercise in preventing NIDDM in people with impaired glucose tolerance. The Da Qing IGT and Diabetes Study. Diabetes Care. 1997; 20: 537-544.

32. Obert J, Pearlman M, Obert L, Chapin S. Popular Weight Loss Strategies: a Review of Four Weight Loss Techniques. Curr Gastroenterol Rep. 2017; 19: 61.

33. Petermann-Rocha $F$, Durán E, Labraña AM, MartínezSanguinetti MA, Leiva AM, Garrido-Méndez A, et al. What is the prevalence of metabolically healthy obesity in Chile? Rev Chil Nutr. 2019; 46: 264-270.

34. Velasco-Estrada A, Orozco-González CN, Zúñiga-Torres G. Association of quality of diet and obesity. PSM. 2018; 16: 132-151.

35. Hosseini SH, Jones JM, Vatanparast H. Association between Grain Intake, Nutrient Intake, and Diet Quality of Canadians: Evidence from the Canadian Community Health SurveyNutrition 2015. Nutrients. 2019; 11: 1937.

36. Olivares S, Zacarías I, González CG. Motivations and barriers of chilean children; threats or opportunities for the implementation of 2013 food based dietary guidelines? Nutr Hosp. 2014; 30: 260-266.

37. Herforth A, Arimond M, Álvarez-Sánchez C, Coates J, Christianson K, Muehlhoff E. A Global Review of Food-Based Dietary Guidelines. Adv Nutr. 2019; 10: 590-605.

38. Mathe N, Agborsangaya CB, Loitz CC, Johnson JA, Johnson ST. HealthBehaviours and Awareness of Canada's Food Guide: A Population-based Study. Can J Diet Pract Res. 2016; 77: 66-71.

39. Olivares-Garrido M, Chavez-Mora E. Using a social media network as a strategy to promote healthy eating in adolescents. RCIM. 2019; 11:1 13-124.

40. Rodriguez-Osiac L, Pizarro-Quevedo T. Law of Food Labelling and Advertising: Chile innovating in public nutrition once again. Rev Chil Pediatr. 2018; 89: 579-581. 